${ }^{1}$ A Uusküla, ${ }^{1} \mathrm{M}$ Raag, ${ }^{2} \mathrm{~K}$ Abel-Ollo, ${ }^{2} \mathrm{~A}$ Talu, ${ }^{2} \mathrm{~K}$ Rüütel, ${ }^{2} \mathrm{M}$ Salekesin, ${ }^{2} \mathrm{~L}$ Niccolai, ${ }^{3} \mathrm{~T}$ White, ${ }^{3} \mathrm{R}$ Heimer. 'University of Tartu, Tartu, Estonia; ${ }^{2}$ National Institute for Health Development, Tallinn, Estonia; ${ }^{3}$ Yale University School of Public Health, New Haven, CT, United States

Background STI prevalence or incidence data among HIV infected can help to distinguish populations with different levels of sexual HIV transmission risk. Herpes simplex virus type 2 (HSV-2) is considered useful because it is almost exclusively sexually transmitted, cannot be cured and leads to the production of lifelong antibodies. Unfortunately, few IDU studies include HSV-2 prevalence data.

Aim of the current work is to examine prevalence of and associations between HSV-2 infection, and HIV infection among injecting drug users (IDUs) in Kohtla-Järve, Estonia.

Methods Current IDU were recruited using chain referral methodology (RDS). Informed consent was obtained, a structured questionnaire including demographics, drug use history, and sexual risk behaviour was administered, and a blood sample was collected for HIV and HSV-2 antibody testing.

Results A total of 600 subjects were recruited in 2012. Subjects were primarily male (73\%), with a mean age of 30 (SD 4.9) years. The prevalences of HIV and HSV-2 were $62 \%$, and $32 \%$, accordingly. Odds for being HSV-2 positive was higher among HIV infected IDUs (OR $1.9 .95 \%$ CI $1.3-2.9)$. One third (27\%) of the sexually active IDUs reported always using condoms (with in the last 4 weeks) prior the study.

Being HSV-2 positive was not associated with reported injection risk behaviour. HSV-2 seropositivity was associated with gender (higher among women; OR 2.7, 95\% CI 1.8-4.0), and sexual behaviour: higher among those reporting IDU-sexual partners (OR 1.7, $95 \%$ CI 1.1-2.7), and those not always using condom (OR 2.0, 95\% CI 1.2-3.4).

Conclusion HSV-2 seroprevalence can be used as a marker of (long-term) sexual risk. However, it might not capture more recent behaviour change in response to the HIV/AIDS among population groups studied.

High prevalence of HSV2 infection coupled with low reported condom use highlight the need of targeted sexual risk reduction interventions for IDU and their partners.

\section{P3.226 PRE-EXPOSURE PROPHYLAXIS (PREP) IS ESTIMATED TO BE A COST-EFFECTIVE ADDITION TO ANTIRETROVIRAL THERAPY (ART) FOR HIV PREVENTION IN A GENERALISED EPIDEMIC SETTING}

doi:10.1136/sextrans-2013-051184.0683

'R Ying, ' $\mathrm{C}$ Celum, ${ }^{1} \mathrm{~J}$ Baeten, ${ }^{1} \mathrm{P}$ Murnane, ${ }^{1} \mathrm{~T}$ Hong, ${ }^{1} \mathrm{M}$ Krows, ${ }^{2} \mathrm{H}$ van Rooyen, ${ }^{2} \mathrm{H}$ Humphries, 'J P Hughes, ${ }^{1,3} \mathrm{R}$ Barnabas. 'University of Washington, Seattle, WA, United States; ${ }^{2}$ Human Sciences Research Council, Sweetwaters, South Africa; ${ }^{3} F r e d$ Hutchinson Cancer Research Center, Seattle, WA, United States

Background In KwaZulu-Natal, South Africa, young women face an extraordinarily high risk for HIV acquisition, with annual incidence estimates of $6 \%$. ART-based strategies for HIV prevention have the potential to significantly decrease HIV incidence, but the impact of PrEP in addition to ART scale-up is undefined. Modeling studies suggest that PrEP targeted to highest-risk groups could maximise benefits and contain costs.

Methods We developed a deterministic transmission model of HIV that stratifies the population by age, sexual activity, and includes HIV infection stage. The model was parameterized using data from community-based HIV counselling and testing studies in KwaZuluNatal and validated using independent HIV prevalence and incidence estimates.

We estimated the effectiveness and cost-effectiveness of a 'test and treat' scenario, targeted PrEP by age and sexual activity, and general PrEP provision. Each scenario was in addition to anticipated baseline ART scale-up for CD4 350 from 35\% in 2013, as observed in KwaZulu-Natal, to $60 \%$ in 2018 (following national guidelines). We assumed PrEP efficacy of $70 \%$.

Results 'Test and treat' (ART for $80 \%$ of all HIV-positive persons) reduced HIV incidence by $58 \%$ and averted $25 \%$ of cumulative infections by 2025 , at an additional $\$ 39,900$ per infection averted compared to baseline ART scale-up. PrEP targeted to $60 \%$ of 20-29-year-olds, in addition to baseline ART scale-up, reduced incidence by $42 \%$ and averted $22 \%$ of infections at an additional $\$ 22,500$ per infection averted, whereas PrEP targeted to $80 \%$ of high-risk individuals reduced incidence by $33 \%$ and averted $13 \%$ of infections at an additional $\$ 7,400$ per infection averted. PrEP coverage of $20 \%$ of the general population reduced incidence by $37 \%$ and incident infections by $18 \%$, at an additional $\$ 26,900$ per infection averted.

Conclusion In a generalised HIV epidemic setting PrEP is a costeffective addition to ART, with targeted PrEP being more cost-effective than generalised PrEP distribution.

\section{P3.227 A COMPARISON OF MICROSIMULATION AND DETERMINISTIC APPROACHES TO MODELLING OF SEXUALLY TRANSMITTED INFECTION DYNAMICS}

doi:10.1136/sextrans-2013-051184.0684

'L F Johnson, ${ }^{2} \mathrm{~N}$ Geffen. ${ }^{1}$ Centre for Infectious Disease Epidemiology and Research, University of Cape Town, Cape Town, South Africa; ${ }^{2}$ Centre for Social Science Research, University of Cape Town, Cape Town, South Africa

Background Deterministic models are widely used in simulating the potential effect of programmes for the prevention and treatment of HIV and other sexually transmitted infections (STIs). However, most deterministic models are frequency-dependent and do not model pair formation explicitly, which can lead to inaccuracies. We aim to quantify these inaccuracies by comparing a frequency-dependent deterministic model to a 'gold standard' microsimulation model of pair formation.

Methods An individual-based microsimulation model was created to represent as closely as possible the assumptions of a previouslydeveloped deterministic model, which simulates heterosexual transmission of seven different STIs (HIV, genital herpes, syphilis, chancroid, gonorrhoea, chlamydia and trichomoniasis) as well as bacterial vaginosis and vaginal candidiasis, in the South African population. The microsimulation model was extended to simulate pair formation. For each STI, steady-state endemic prevalence levels were estimated using both models.

Results The ratio of the endemic STI prevalence in the microsimulation model to that in the deterministic model varied from 0.88 for HIV to 0.81 for genital herpes, 0.53 for chlamydia, 0.42 for trichomoniasis, 0.12 for gonorrhoea and 0.00 for both syphilis and chancroid. In contrast, the ratio was close to 1 for non-sexually transmitted infections (1.00 for vaginal candidiasis and 1.02 for bacterial vaginosis). The ratio was strongly negatively associated with the fraction of transmission occurring in the first 6 months of infection $(r=-0.98)$.

Conclusion Frequency-dependent deterministic models of STIs tend to exaggerate the levels of transmission in the early stages of infection, because they ignore the period in which individuals remain in contact with the partner who infected them. This bias is particularly significant for non-viral STIs. Further work is required to assess whether microsimulation models of pair formation predict more accurately the effects of STI prevention and treatment programmes.

\section{P3.228 HSV-2 SEROINCIDENCE AND ITS ASSOCIATION WITH MEDICAL MALE CIRCUMCISION, HIV, GENITAL ULCER DISEASE, AND PENILE EPITHELIAL TRAUMA}

doi:10.1136/sextrans-2013-051184.0685 